\title{
Percutaneous transhepatic biliary drainage and stenting for malignant obstructive jaundice: A report of two cases
}

\author{
JIN-HUI SHAO ${ }^{1}$, HAI-XING FANG ${ }^{1}$, GUO-WEI LI ${ }^{1}$, JIA-SHENG HE ${ }^{1}$, BAO-QUAN WANG $^{2}$ and JUN-HUI SUN ${ }^{2}$ \\ ${ }^{1}$ Department of Hepatobiliary Surgery, Fuyang People's Hospital, Hangzhou, Zhejiang 236000; \\ ${ }^{2}$ Division of Hepatobiliary and Pancreatic Surgery, Department of Surgery, First Affiliated Hospital, \\ School of Medicine, Zhejiang University, Hangzhou, Zhejiang 310003, P.R. China
}

Received December 30, 2014; Accepted July 13, 2015

DOI: $10.3892 /$ etm.2015.2701

\begin{abstract}
Malignant obstructive jaundice comprises a group of diseases that can be caused by primary biliary and extra-biliary carcinomas. Generally, surgical resection is the primary treatment for malignant obstructive jaundice; however, for the patients that are unable to undergo surgery, urgent treatment is required to improve hepatic function. Percutaneous transhepatic biliary drainage (PTBD) and stenting are emerging alternative treatments for malignant obstructive jaundice. PTBD and stenting have exhibited good efficacy for the treatment of malignant obstructive jaundice, with few complications and reduced associated pain.
\end{abstract}

\section{Introduction}

Malignant obstructive jaundice comprises a group of diseases that can be caused by primary biliary carcinomas, such as cholangiocarcinoma and gallbladder cancer, and extra-biliary carcinomas, such as ampullary, pancreatic and gastric cancer and hepatocellular carcinoma (1). Malignant obstructive jaundice can lead to hyperbilirubinemia, anorexia, pruritus, cholangitis, septicemia and liver failure. Generally, surgical resection remains the primary treatment for malignant obstructive jaundice; however, in numerous cases the malignant obstructive jaundice is detected when the disease is already at an advanced stage (2). For the patients that are unable to undergo surgery, urgent treatment is required to improve hepatic function, in order to facilitate the addition of subsequent anti-tumor therapy to their treatment regimen. The average survival time of patients with obstructive jaundice is $<3$ months (3). Percutaneous transhepatic biliary drainage

Correspondence to: Professor Jun-Hui Sun, Division of Hepatobiliary and Pancreatic Surgery, Department of Surgery, First Affiliated Hospital, School of Medicine, Zhejiang University, 79 Qingchun Road, Hangzhou, Zhejiang 310003, P.R. China E-mail: sunjh121@163.com

Key words: percutaneous transhepatic biliary drainage, malignant obstructive jaundice, stenting
(PTBD) and stenting are emerging alternative treatments for malignant obstructive jaundice that exhibit good clinical efficacy and few complications and lead to limited patient suffering. PTBD and stenting is the first choice for elderly patients, patients with inoperable malignant obstructive jaundice, and patients with postoperative recurrence, diabetes, or cardiovascular disease.

\section{Case report}

Case 1. The patient was a 51-year-old man who presented with $\sim 3$ weeks' history of obstructive jaundice. The patient was admitted to Fuyan People's Hospital (Fuyang, China) on June 12, 2014. Liver and kidney function analyses, and routine blood tests were conducted upon admission; and heaptic bilirubin levels were higher than normal. Abdominal computed tomography (CT) showed pancreatic cancer, as well as dilation of the intra- and extrahepatic bile ducts. The patient was at an advanced disease stage and refused to undergo surgical resection. PTBD and stenting were performed in the interventional operating room following the provision of informed consent by the patient. The procedure to puncture the biliary duct was guided under ultrasound (US). An 18-G Chiba needle and a 0.035 -inch guide-wire were used to gain access to the biliary system using a right-sided puncture approach. An 8-F sheath was inserted to facilitate the procedure. A 0.035-inch hydrophilic guide-wire was advanced through the stenosis into the duodenum. The hydrophilic guide-wire was exchanged for a 0.035 -inch stiff guide-wire. Finally, a self-expanding metallic stent [Micro-Tech (Nanjing) Co., Ltd.] was inserted alongside the guide-wire into the narrow segment (Fig. 1). A CT scan was performed a month after the PTBD and stenting (Fig. 2), and the patient recovered well following the surgery.

Case 2. The patient was an 84-year-old man who presented with $\sim 1$ week's history of obstructive jaundice. The patient also presented with symptoms of pruritus. The patient was admitted to Fuyan People's Hospital on August 16, 2014. Liver and blood coagulation function analyses, and routine blood tests were conducted upon admission. Abdominal CT was used to diagnose carcinoma of the ampulla of Vater, with extrahepatic bile duct dilatation. The patient's condition 


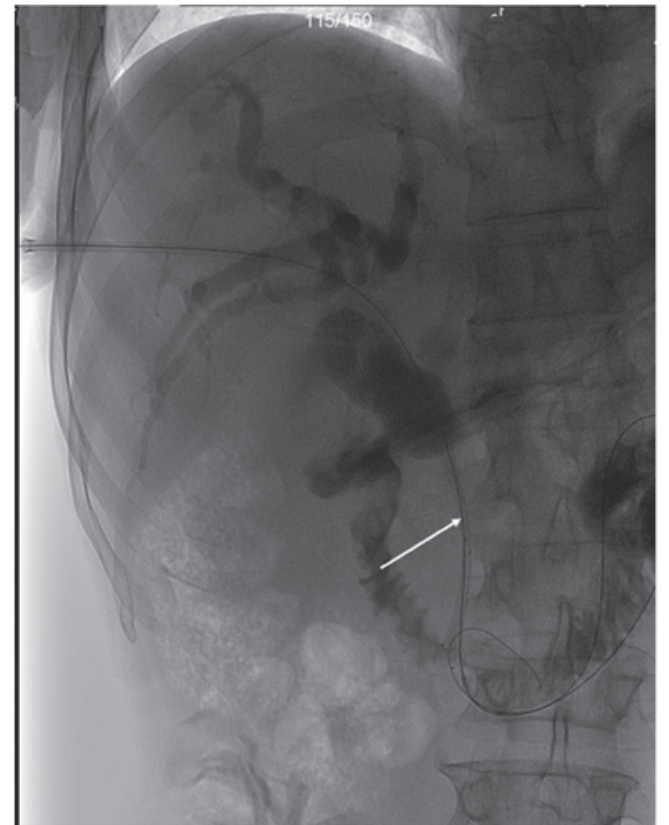

Figure 1. Cholangiography revealed that the common bile duct stricture in the lower section measured $\sim 5 \mathrm{~cm}$. The minimally invasive implantation of the $8-\mathrm{F} / 60 \times 10 \mathrm{~mm}$ metal stent is shown by the arrow.

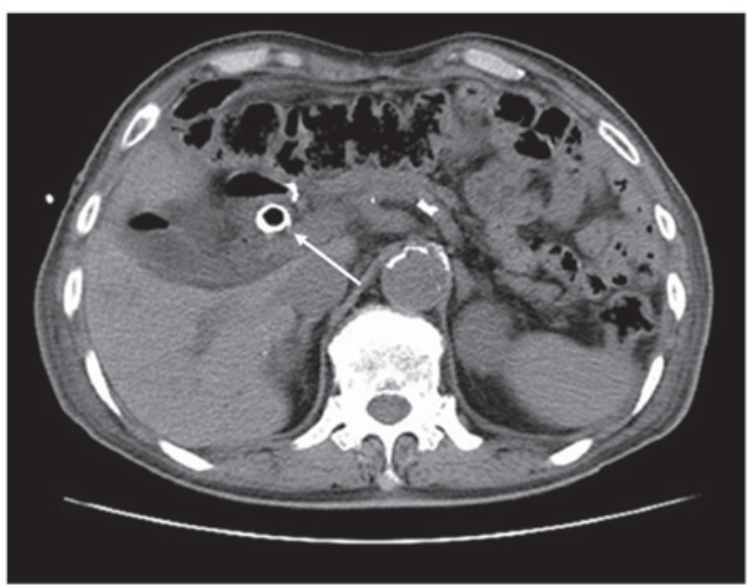

Figure 2. Computed tomography performed 1 month after percutaneous transhepatic biliary drainage and stenting. The high-density circle is the biliary stent (arrows). The image showed there was no bile duct dilatation, which indicated that the biliary tract was unobstructed.

was too advanced for surgery to be advisable. PTBD and stenting were performed to resolve the obstructive jaundice. Cholangiography revealed common bile duct stenosis in the lower section of the bile duct, which measured $\sim 6 \mathrm{~cm}$ (Fig. 3). A minimally invasive implantation of an $8-\mathrm{F} / 80 \times 10 \mathrm{~mm}$ metal stent was performed. The biliary stent was implanted successfully to cover the stenotic bile duct (Figs. 4 and 5). The patient experienced an uneventful post-procedural outcome. A further CT scan was performed 14 days after PTBD and stenting (Fig. 6), which showed that the metal stent was at the correct location, and no bile duct dilatation was observed.

PTBD and stenting procedure. The PTBD and stenting were performed with US-guided and digital subtraction

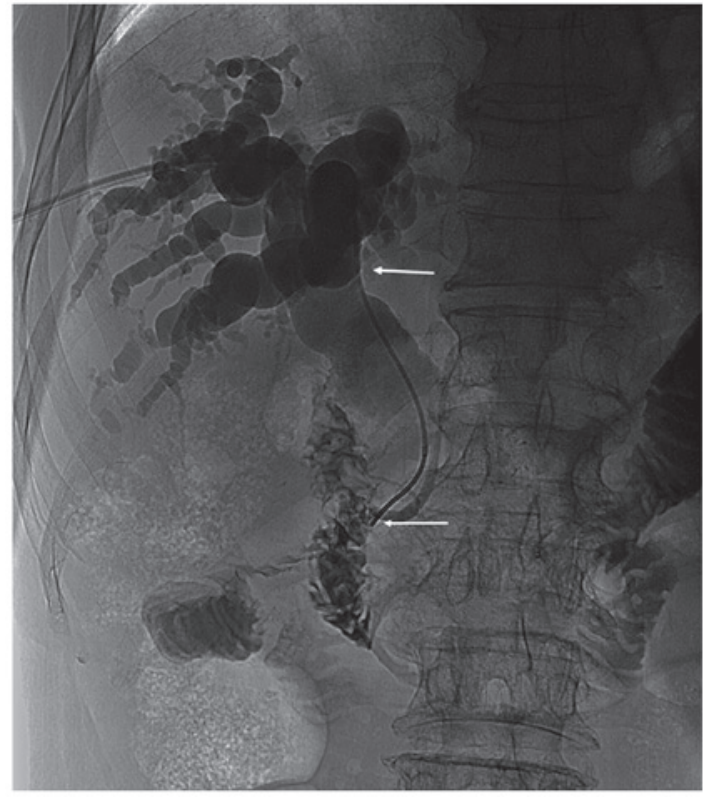

Figure 3. Following the injection of the contrast agent, it was observed that the lower segment of the common bile duct was narrow. The length of the narrow segment was $\sim 6 \mathrm{~cm}$ (the distance between the two arrows).

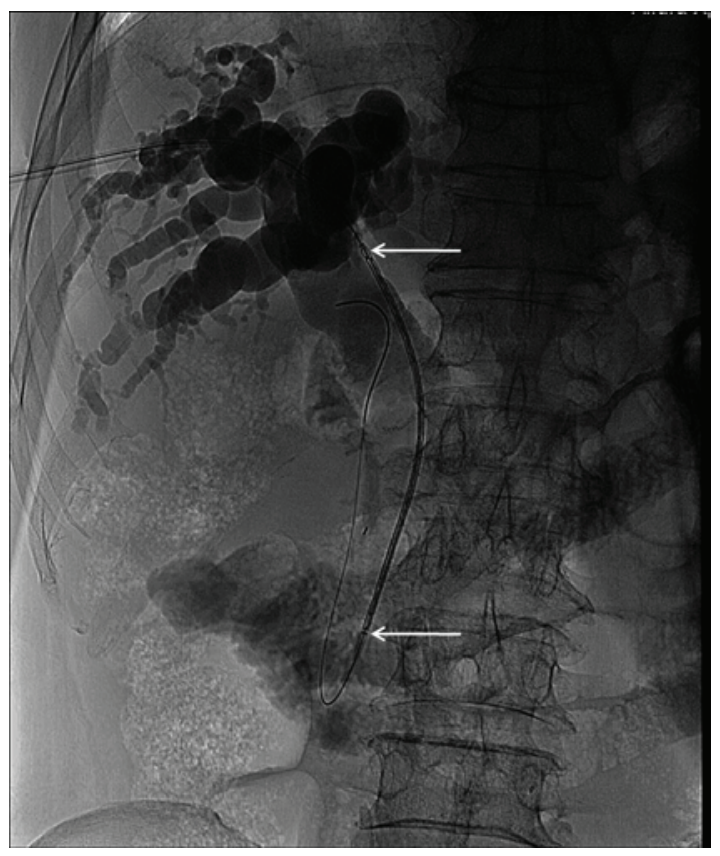

Figure 4. Fluoroscopy showed that the guide-wire being circled in the lumen of the diverticulum and metal stent (between the two arrowheads) passed through the orifice of the diverticulum into the distal segment of the duodenum during the percutaneous transhepatic biliary drainage and stenting procedure.

angiography. Cholangiography was first used to evaluate the exact level and extension of the stenosis or stenoses and to select the most appropriate liver segments for drainage. Finally, a self-expanding metallic stent [Micro-Tech (Nanjing) Co., Ltd., Nanjing, China] was inserted alongside the guide-wire into the narrow segment.

All patients were monitored for vital signs, oxygen saturation, and liver and kidney function post-surgery, and 
Table I. Liver function of the patients before and after PTBD and stenting.

\begin{tabular}{|c|c|c|c|c|c|c|}
\hline \multirow[b]{2}{*}{ Case no. } & \multicolumn{2}{|c|}{ Total bilirubin $(\mu \mathrm{mol} / \mathrm{l})$} & \multicolumn{2}{|c|}{ Direct bilirubin $(\mu \mathrm{mol} / \mathrm{l})$} & \multicolumn{2}{|c|}{$\operatorname{ALT}(\mathrm{U} / \mathrm{l})$} \\
\hline & Before & After & Before & After & Before & After \\
\hline 1 & 333.59 & 106.59 & 229.90 & 71.00 & 196 & 93 \\
\hline 2 & 419.02 & 77.75 & 278.20 & 48.30 & 161 & 27 \\
\hline
\end{tabular}

PTBD, percutaneous transhepatic biliary drainage; ALT, alanine transaminase; Before, 1 day before PTBD and stenting; After, 1 month after PTBD and stenting.

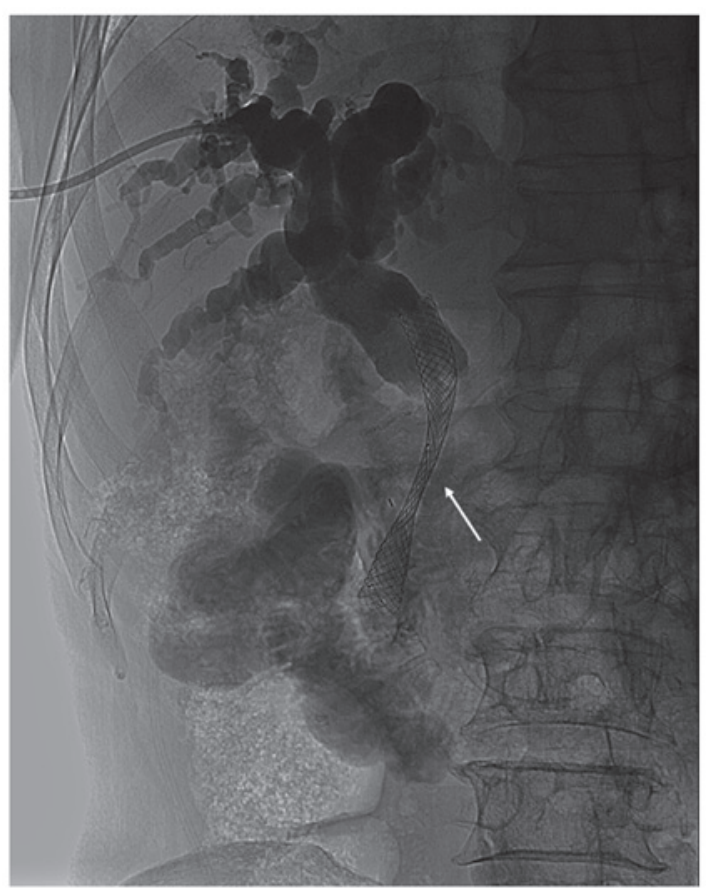

Figure 5. Cholangiography revealed contrast agent passing through the stent into the duodenum. The image shows that the biliary stent was successfully expanded (arrow).

routine blood investigations were performed. Rehydration was considered paramount to protect the liver and prevent infection.

Results. The PTBD and stenting procedures in the two patients were considered successful and were performed without the occurrence of procedure-associated complications. The patients were followed-up for 1 month post-surgery. The patients' liver function post-procedure (1 month after PTBD and stenting) was notably improved compared with the pre-procedure (1 day before PTBD and stenting) liver function (Table I). In addition, the patients' jaundice was evidently improved. The present study indicates that PTBD and stenting can exert a definitive positive effect on hepatic function in patients with obstructive jaundice.

\section{Discussion}

Malignant biliary obstruction may be caused by cholangiocarnon biliary carcinomas (1-3). The current treatment options for malignant obstructive jaundice include surgical, interventional therapy and endoscopic therapy. Surgery is primarily applied

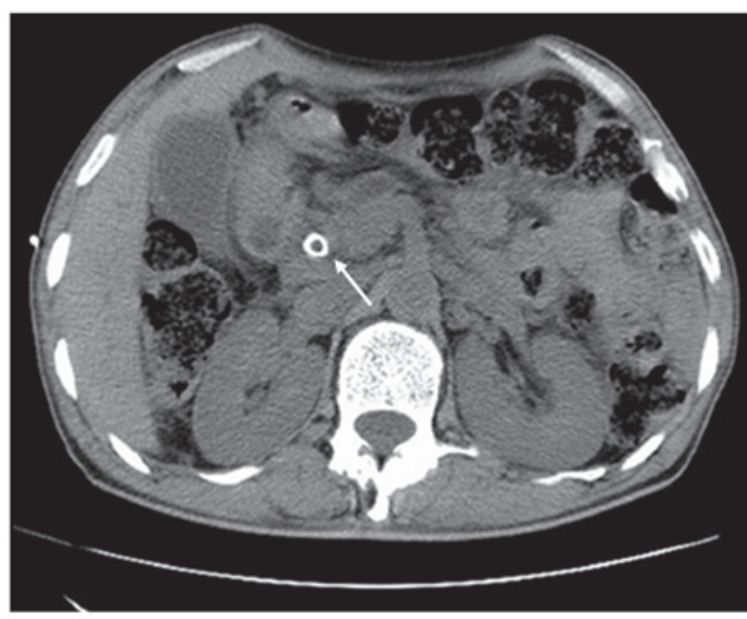

Figure 6. Computed tomography (CT) performed 14 days after percutaneous transhepatic biliary drainage and stenting. The arrow indicates the unobstructed biliary stent. The CT scan revealed no obvious bile duct dilatation.

in cases of extrahepatic bile duct obstruction. Surgery may be used to relieve jaundice in addition to removing lesions and peripheral vascular invasion (1). The patients with a generally poor condition, diabetes, cardiovascular disease complications or the elderly may not be suitable to undergo surgery. Interventional therapy is the preferred treatment for the patients with the aforementioned conditions (3). At the time of diagnosis, $90 \%$ of patients with malignant obstructive jaundice may benefit from palliative treatment only (4). The objective of palliation is to relieve jaundice-related symptoms, preventing cholangitis. In recent years, with the development of technology and medical materials, PTBD and stenting have exhibited good efficacy, with few complications and reduced associated pain, and are emerging as crucial palliative treatments for malignant obstructive jaundice $(5,6)$. In addition to prolonging patient survival time, PTBD and stenting are able to improve quality of life. In 1981, Fletcher et al first reported three cases of patients with malignant obstructive jaundice that deposition ${ }^{199}$ Ir into biliary via a PTCD drainage tube (7). The approach achieved good results in controlling intraductal tumor growth. However, there were a number of treatment deficiencies. As ${ }^{199} \mathrm{Ir}$ is a high source of radiation, and safe levels of human exposure to radiation dose are limited, resulting in patients received a discontinuous radiation therapy with long-term drainage tube carrying. The primary complications associated with PTBD and stenting include the following: i) Drainage tube or biliary stent dislocation; ii) cholangitis; iii) hemobilia; and 
iv) pancreatitis $(4,5)$. No complications were observed in the cases described in the present study. In conclusion, PTBD and stenting are able to markedly improve hepatic function, without serious complications, and thus represent a potentially safe and feasible intervention for patients with malignant obstructive jaundice.

\section{Acknowledgements}

This study was supported by the Science and Technology Development Plan of Fuyang City (no. 2014SK013).

\section{References}

1. Barauskas G, Gulbinas A and Pundzius J: Results of surgical treatment of carcinoma of papilla of Vater. Medicina (Kaunas) 43: 455-462, 2007.
2. Brountzos EN, Ptochis N, Panagiotou I, Malagari K, Tzavara C and Kelekis D: A survival analysis of patients with malignant biliary strictures treated by percutaneous metallic stenting. Cardiovasc Intervent Radiol 30: 66-73, 2007.

3. van Delden OM and Lameris JS: Percutaneous drainage and stenting for palliation of malignant bile duct obstruction. Eur Radiol 18: 448-456, 2008.

4. Qian XJ, Zhai RY, Dai DK, Yu P and Gao L: Treatment of malignant biliary obstruction by combined percutaneous transhepatic biliary drainage with local tumor treatment. World J Gastroentero1 12: 331-335, 2006.

5. Feng GH, Cai Y, Jia Z, Yang DQ, Chen H, Jin HC, Yu QH, Zhu W and Wang CX: Interventional therapy of malignant obstructive jaundice. Hepatobiliary Pancreat Dis Int 2: 300-302, 2003.

6. Garcarek J, Kurcz J, Guziński M, Janczak D and Sasiadek M: Ten years single center experience in percutaneous transhepatic decompression of biliary tree in patients with malignant obstructive jaundice. Adv Clin Exp Med 21: 621-632, 2012.

7. Fletcher MS, Brinkley D, Dawson JL, Nunnerley H, Wheeler PG and Williams R: Treatment of high bileduct carcinoma by internal radiotherapy with iridium-192 wire. Lancet 2: 172-174, 1981. 